\title{
Multimodal Stimulation System to Control Fibroblast Proliferation Using Optical and Ultrasonic Stimulation ${ }^{+}$
}

\author{
Da-won An and Se-woon Choe * \\ Department of Medical IT Convergence Engineering, Kumoh National Institute of Technology, 350-27 Gumi- \\ daero, Gumi, Gyeongbuk 39253, Korea; 20170667@kumoh.ac.kr \\ * Correspondence: sewoon@kumoh.ac.kr; Tel.: +82-54-478-7781 \\ + Presented at the 7th International Electronic Conference on Sensors and Applications, 15-30 November \\ 2020; Available online: https://ecsa-7.sciforum.net/.
}

Published: 15 November 2020

\begin{abstract}
An optical stimulation shows various effects for skin regeneration and wound treatment by using different wavelength. Similarly, ultrasound stimulation can improve skin wrinkles and contours by inducing the contraction and synthesis of collagen to reduce local fat accumulation. In this study, using commercially available LEDs for skin regeneration masks $(415 \mathrm{~nm}, 630 \mathrm{~nm}, 850$ $\mathrm{nm}$ ), a single wavelength and multiple wavelengths were applied to fibroblast cells in various ways to control the proliferation effect of skin cells. In addition, ultrasonic stimulation was applied simultaneously to quantitatively evaluate the proliferation effect of fibroblasts. As a result, it was confirmed that there was an effect on fibroblast cell proliferation when the LED light stimulation of a specific wavelength was applied, and also the proliferation activity of skin cells increased even in the multimodal stimulation by applying a combination of LEDs and ultrasound.
\end{abstract}

Keywords: LED; fibroblast; ultrasound; CCD-986sk; skin cell; cell proliferation

\section{Introduction}

In recent years, interest in personal skincare systems has been increasing as the population ages and the number of SNS user increases. In addition, there is an increasing number of users of skin care systems that can be proactively used in individual preferred locations to save time and improve their appearance at reasonable cost. Light sources such as lasers and LED (light-emitting diode) are used as stimulants to promote activation of skin cells among the components of the skin care system. There are limitations that laser, which are relatively expensive and inconvenient to use, can cause thermal destruction of effective tissues due to the high heat generated, so LED that are relatively low cost among various light stimuli and have a high degree of freedom of choice are widely used [1,2]. For this reason, related research is actively conducted to quantitatively evaluate skin regeneration effects using LEDs. For example, the effect on skin cells by LED varies depending on the light characteristics such as exposure light intensity, wavelength, and exposure time [3,4]. A single stimulation system by LED for skincare has been actively studied. However, complex wavelengths or non-invasive fusion stimulation needs further application. Therefore, in this paper, LEDs and ultrasound (US) for commercially available skin regeneration mask were applied to develop a multimodal stimulation system capable of applying to fibroblasts of a particular location. After conducting a simultaneous application experiment with the sole application of light stimulation and ultrasonic stimulation, the images were obtained through a microscope to show its significance. 


\section{Methods}

To evaluate the performance of the stimulation system, the fibroblasts cells (CCD-986sk cells, Korean Cell Line Bank, Korea) were used and incubated at $37^{\circ} \mathrm{C}$ humidified incubator with $5 \% \mathrm{CO}_{2}$ for $72 \mathrm{~h}$. The cells were cultured in a medium solution consisting of IMDM (Isocove's Modified Dulbecco's Medium), 10\% FBS (PH 7.4) and 1\% Penicillin Streptomycin. For comparison of cell proliferation effect, experimental groups were designed as follows; LED stimulation group with various wavelengths $(n=17)$, US and LED simultaneous stimulation group $(n=17)$ and control group without stimulation $(n=20)$. LED stimulation systems used various LEDs including red $(R, 630 \mathrm{~nm})$, blue (B, $415 \mathrm{~nm})$, and infrared (IR, $850 \mathrm{~nm}$ ) wavelengths, which are mainly used in conventional LED masks. LED stimulation group was investigated by dividing the total three groups (Group A (R), B $(I R+R+B), C(I R+R))$. Multimodal stimulation groups were divided into three groups (Group D (R+ US), E (IR + R + B + US), F (IR + R + R + US) to apply the LED and US stimulation at the same time in the same position. 3D printer (Cubicon DP-310F, Korea Cubicon Co., Ltd.) was used to make a LED stimulation supporter so that cells can be stimulated in the center of each well of the plate. Ultrasonic stimulation system is configured to maintain a frequency of $10 \mathrm{MHz}$ in waveform generator (Function generator, Tecktronics Inc., Beaverton, OR, USA) and generates a signal of 100 cycle with an output voltage of up to $20 \mathrm{mV}_{\mathrm{pp}}$ amplifier (75A250A, American Research Corp., Souderton, PA, USA) through ultrasonic probe (V303, Olympus., Japan). Ultrasonic stimulation system was placed at the top of the culture flask where the cells are cultured. It was fixed position to enable stimulation of the cell culture medium surface and simultaneous application with the optical stimulation applied to the bottom of the cell culture flask as in Figure 1.

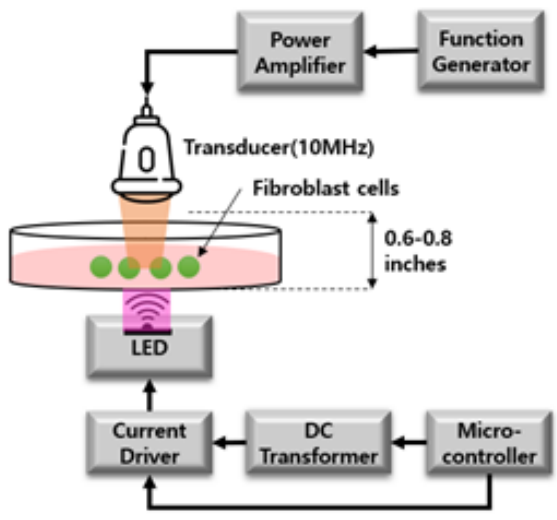

(a)

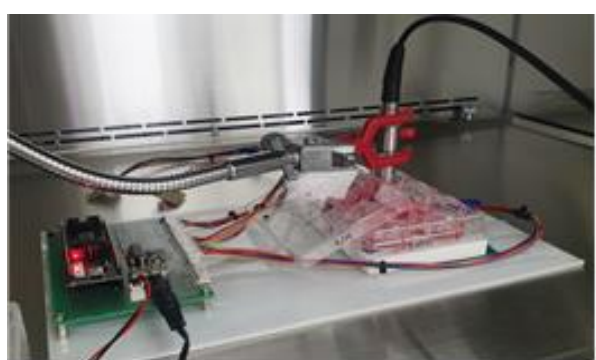

(b)

Figure 1. (a) Conceptual diagram of multimodal stimulation system; (b) Experimental setup for multimodal stimulation system using LED and ultrasound probe.

Cell images were acquired with a reverse optical microscope (Inverted fluorescent microscope, IX73, Olympus, Japan) before and after the stimulation. The images from the same location of the cell culture flask were possible to perform quantitative analysis. Various image processing tools were applied for analysis using MATLAB (MathWorks, Natick, MA, USA) program. After quantitative analysis of the area of the cells, the average increment was compared to the area of the flask.

\section{Results and Discussions}

The representative images of the control group without stimulation, the experimental group (Group $\mathrm{A} \sim \mathrm{C}$ ) applied only LED single stimulus and the multimodal experimental group (Group D F) applied with ultrasonic stimulation are shown in Figure 2. Representative images of the experimental group and the control group were obtained after stimulation application through an optical microscope. Cell growth rate by each group was compared based on the type of stimulation and date of stimulation. Compared to the control group without stimulation, all of the experimental groups 
applied to a single stimulus or multimodal stimulation can be confirmed that the cell differentiation is actively conducted. The average increment of cell proliferation calculated by group is shown in Figure 2.

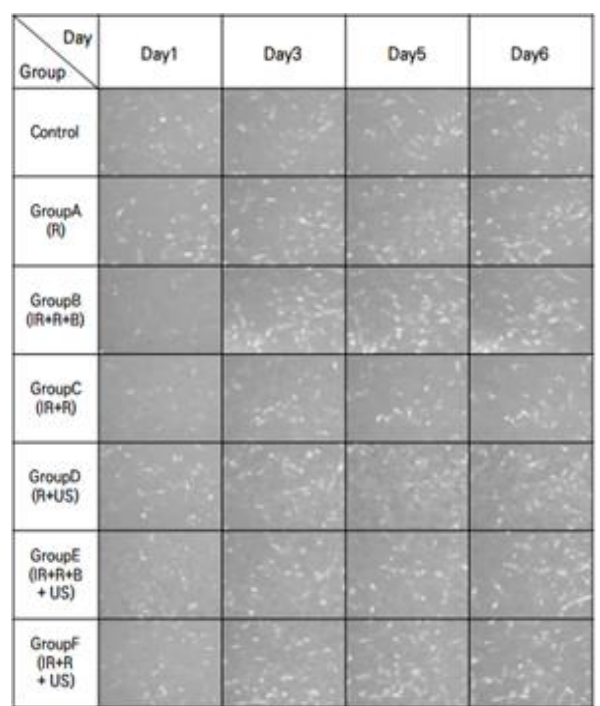

(a)

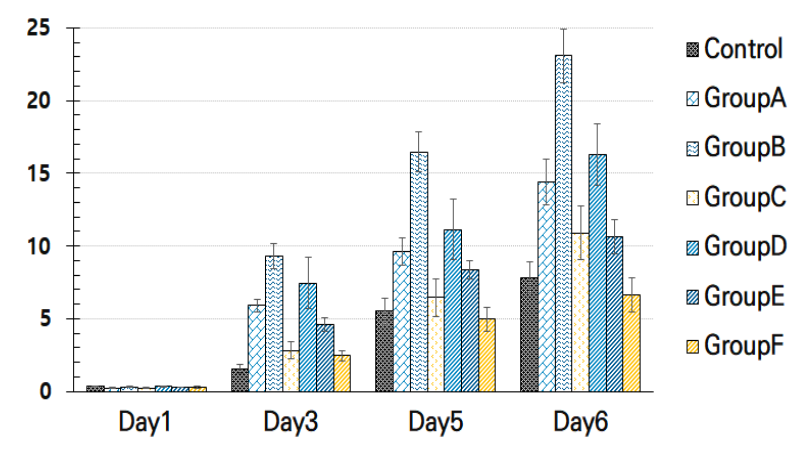

(b)

Figure 2. (a) Representative images of fibroblast cells per group; (b) Experimental results of the cell density with various stimuli.

As a result, the experimental group given a single light stimulation and multimodal stimulation was higher than the control group. Especially, LED stimulation of the complex wavelength of the IR $+\mathrm{R}+\mathrm{B}($ Group $\mathrm{B})$ showed a higher proliferation rate than the stimulation applied to the $\mathrm{R}$ wavelength (Group A) and R + IR wavelength (Group C). Therefore, it was confirmed that the Group B (IR + R + B) applied with blue wavelength shows the most active cell proliferation. On the other hand, in the case of multimodal stimulation to apply LED and ultrasonic stimulation at the same time, the stimulation of Group D ( + US) showed a higher proliferation rate than other stimuli. In addition, it was shown that blue wavelength with ultrasonic stimulation induces higher cell differentiation when applied together.

\section{Conclusions}

In this study, the multimodal stimulation system using LED and ultrasound probe was developed to control the cell proliferation and quantitatively analyzed its effects. In order to verify the effect of optical stimulation, a single or complex wavelengths and ultrasonic stimulation were applied. All experimental groups applied to LED and multimodal stimulation showed excellent cell proliferation rate compared to the control group without stimulation. Among the LED stimulation experiments, the cell proliferation was most actively proliferation in Group B (IR $+R+B)$ applied with blue wavelength. In the case of multimodal stimulation to apply LED and US stimulation at the same time, the stimulation of Group D ( + US) showed a higher proliferation rate than other stimuli. In order to increase the cell proliferation rate using LED stimulation, it is better to use a composite light stimulus containing blue wavelengths than a single light stimulus. For multimodal stimulators using additional ultrasound probe, higher cell proliferation rate was observed when it comes with a composite wavelength than a single $\mathrm{R}$ wavelength.

Author Contributions: Conceptualization, S.-w.C.; methodology, S.-w.C.; software, S.-w.C.; validation, S.-w.C. and D.-w.A.; formal analysis, S.-w.C. and D.-w.A.; writing-original draft preparation, S.-w.C. and D.-w.A.; writing - review and editing, S.-w.C.; supervision, S.-w.C. project administration, S.-w.C.; funding acquisition, S.-w.C. All authors have read and agreed to the published version of the manuscript. 
Funding: This research was supported by Basic Science Research Program through the National Research Foundation of Korea (NRF) funded by the Ministry of Education (NRF-2019R1F1A1062397).

Conflicts of Interest: The authors declare no conflict of interest.

\section{References}

1. Greaves, A.J. The effects of narrowbands of visible light upon some skin disorders: A review. Int. J. Cosmet. Sci. 2016, 38, 325-345.

2. Trelles, M.A. Phototherapy in anti-aging and its photobiologic basics: A new approach to skin rejuvenation. J. Cosmet. Dermatol. 2006, 5, 87-91.

3. Papageorgiou, P.; Katsambas, A.; Chu, A. Phototherapy with blue (415 nm) and red (660 nm) light in the treatment of acne vulgaris. Br. J. Dermatol. 2000, 142, 973-978.

4. Lee, S.Y.; You, C.E.; Park, M.Y. Blue and Red Light Combination LED Phototherapy for Acne Vulgaris in Patients with Skin Phototype IV. Lasers Surg. Med. 2007, 39, 180-188.

Publisher's Note: MDPI stays neutral with regard to jurisdictional claims in published maps and institutional affiliations.

(C) 2020 by the authors. Submitted for possible open access publication under the terms and conditions of the Creative Commons Attribution (CC BY) license (http://creativecommons.org/licenses/by/4.0/). 